\title{
¿Aptitudes y qué más? \\ Principios para las pedagogías de praxis en la educación superior
}

Melanie Walker ${ }^{\star}$

\begin{abstract}
Resumen
Las universidades tienen como funciones la investigación, la enseñanza, la educación profesional y varias formas de compromiso público (incluidos el intercambio de conocimientos y las asociaciones). Esto nos proporciona poca información para pensar en justicia, y tampoco nos indica ningún punto de vista normativo acerca de los propósitos de la educación superior, que debería informar de la política educativa, las prácticas institucionales, los procesos pedagógicos y las relaciones globales. La educación superior debería estar comprometida con lo que Habermas describe como las "emergencias" morales de nuestra era en todo el mundo. ¿A quién deben justicia los graduados y las instituciones de la educación secundaria?
\end{abstract}

\section{Palabras clave}

Universidad, educación superior, pedagogías de la praxis, derechos pedagógicos, evaluación, justicia social.

\begin{abstract}
The functions of the universities are research, education, vocational education and various forms of public engagement (including the exchange of knowledge and partnerships). This provides little information to think about justice, nor do indicate a regulatory point of view about the purposes of higher education, which should inform about education policy, institutional practices, pedagogical processes and global relations. Higher education should be committed to what Habermas describes as moral "emergencies" of our era in the world. Who to justice graduates and the institutions of secondary education?
\end{abstract}

\section{Key words}

University, pedagogies of praxis, educational rights, evaluation, social justice.

Forma sugerida de citar: Walker, Melanie. 2011. “Aptitudes y qué más? Principios para las pedagogías de praxis en la educación superior”. Universitas 15. Julio/ Diciembre. Pp. 85-107.

Facultad de Educación de la Universidad de Nottingham, Reino Unido. E-mail: melanie.walker@nottingham.ac.uk Ponencia presentada en el IV Congreso de Universidad, Cooperación al Desarrollo en BellaterraBarcelona, 12-14 de noviembre de 2008. 


\section{Introducción}

Paolo Blasi escribe que el reto de la sociedad europea actual es ir más allá de la "sociedad del conocimiento" y evolucionar en lo que se podría identificar como "la sociedad de la sabiduría". El conocimiento es el uso consciente de la información; "sabiduría" significa escoger el propio comportamiento basándose en el conocimiento y los valores compartidos para mejorar el bienestar de todos, y la conciencia de que las acciones personales tienen consecuencias sociales (citado en Marga, 2008: 117).

Mi preocupación se centra específicamente en lo que esto significa para los objetivos humanísticos y culturales de las universidades, y particularmente en los procesos pedagógicos y la adquisición y la construcción dialógica del conocimiento por parte de los estudiantes universitarios en un entorno de investigación. Lo que los estudiantes pueden conocer, aquello en lo que pueden convertirse y llegar a ser a través de la educación superior es un proyecto de gran importancia para sí mismos, para la sociedad y para todo el mundo; a la vez, también es significativo el hecho de que estudiantes diferentes tengan las mismas oportunidades a través del diseño justo de los acuerdos pedagógicos para llegar a ser lo que valoran.

Lo que se necesita es una atención explícita a las pedagogías del desarrollo humano y para éste, definido por el programa de desarrollo de las Naciones Unidas como "creación de un entorno en el que las personas puedan desarrollar su potencial completo y dirigir vidas productivas y creativas de acuerdo con sus necesidades y sus intereses". El objetivo normativo debería ser que la educación superior contribuyera sustancialmente en la calidad de vida y el bienestar individual y social, además de mejorar las vidas y las sociedades humanas a través de la formación de las capacidades humanas para ser y hacer lo que las personas valoran; ser y hacer para tener una buena vida, ejercer elecciones genuinas y participar en la toma de decisiones igualitarias que afecten sus vidas. Se entiende que el objetivo del desarrollo humano es la "libertad" para ejercer elecciones genuinas y participar en la toma de decisiones que afecten en la vida de las personas. Esto se ve reforzado por los derechos humanos, que ayudan a garantizar el bienestar y la dignidad de todos, creando el respeto por sí mismo y por los otros. El hecho de emprender el desarrollo humano y social también 
significa orientar las universidades a las preocupaciones y las obligaciones del bienestar humano, y no solo al crecimiento económico a cualquier precio.

Por consiguiente, expongo tres principios centrales para las pedagogías de praxis que son transformativas, críticas y atentas, tanto para los conocimientos como para las acciones responsables que se llevan a término en la sociedad. Unas pedagogías de praxis que estarían determinadas por una versión "fuerte" de equidad, que pretende reequilibrar el desarrollo personal, las oportunidades económicas y los objetivos sociales, que fomenta la equidad, que conecta las universidades con la sociedad y que promueve una amplia variedad de finalidades en sociedades pluralistas y democráticas. Las pedagogías de praxis estarían determinadas por dos principios básicos: que todas las personas tienen el mismo valor moral y la misma dignidad (Nussbaum, 2000) y que lo que se consigue en la educación importa individualmente y socialmente para hacer que las vidas humanas sean mejores.

\section{Objetivos de la educación superior}

En primer lugar, trataré de los objetivos de la educación superior que determinan el contexto y el entorno en el que tienen lugar la enseñanza y la pedagogía. En una conferencia plenaria reciente, Brighouse (2007) hizo explícita la cuestión importante. Argumentó que necesitamos un nuevo informe normativo de la educación superior, uno que pregunte: “¿A qué intereses sirve la educación superior?" y, evidentemente, la respuesta es a los intereses del cual se promuevan mediante acuerdos pedagógicos en la educación superior. En su ponencia principal en la conferencia sobre Capacidades y desarrollo humano de 2007, Martha Nussbaum (2008) contó una sorprendente anécdota que captura una gran parte de lo que es incorrecto en las intenciones y la dirección de la educación superior hoy en día, como mínimo en el Reino Unido. La autora narra su visita guiada en un templo hindú en Illinois, con un guía joven que había llegado poco antes a EE. UU. procedente de la provincia de Gujarat, en la India. Mientras le mostraba el templo, le contaba sus propias creencias en los poderes espirituales de Pramukh Swami, el actual jefe de la secta hindú Swaminarayan, que según Nussbaum se caracteriza por una obediencia sin sentido crítico al líder, voz del cual se le considera la voz directa de Dios. Nussbaum 
cuenta como el joven señaló el techo del templo y le preguntó si sabía por qué brillaba. Sigue: "Le dije que no lo sabía y, en confianza, esperaba una explicación que invocara los poderes espirituales de Pramukh Swami. Mi guía sonrió anchamente. 'Cables de fibra óptica', dijo. 'Somos los primeros en utilizar esta tecnología en un templo." "Ya lo veis", escribe Nussbaum, "esto es lo que puede destruir fácilmente la democracia: una combinación de sofisticación tecnológica y docilidad total" (ibíd.: 370).

Los últimos años, parece que la educación superior ha estado más interesada en la ciencia y la tecnología y en su aplicación. Hasta las artes creativas se han refundido en las industrias creativas en la campaña para ampliar nuestras economías del conocimiento y aumentar el posicionamiento competitivo en el mercado.

A nivel nacional e internacional, la educación superior se ve como algo central para la creación de la capacidad intelectual y la construcción de los conocimientos y las capacidades para la participación en una economía mundial basada cada vez más en los conocimientos (Castells, 2004; Kenway et al., 2004; Kwiek, 2002; Lauden et al., 2006; Jonathan, 2006; Mclean, 2006; Newman, et al., 2004; Teferra y Altbach, 2003; UNESCO, 1998; Walker, 2006; Banco Mundial, 2002). Castells (2004) sostiene que si el conocimiento es la "lectricidad" de la nueva economía internacional, las instituciones de educación superior son las fuentes de energía de las que debe depender un nuevo proceso de desarrollo.

Las políticas de educación superior se han centrado en los resultados educativos que apoyan el crecimiento económico y los mejores ingresos individuales: el planteamiento de capital humano sobre la educación, que mide los resultados para la educación y aplica un análisis del coste-beneficio a las decisiones sobre gastos y rentabilidad de la educación. La asunción es que el desarrollo y el crecimiento económico significan lo mismo y que ambos se equiparan con el bienestar individual y colectivo.

No es nada sorprendente que el aspecto que más preocupa a los gobiernos sea el hecho de que la educación debería proporcionar a los graduados los conocimientos y las capacidades para participar en la economía. Pero el problema surge cuando hay una variable y para algunos el valor social que supone obtener un título universitario está en declive; cuando no se habla del valor de las oportunidades económicas y de la participación total; y cuando se pasan de 
largo los objetivos como el desarrollo individual y la ciudadanía democrática y los bienes sociales más amplios. La prueba que empieza a prosperar entre los economistas es que el hecho de doblar el PIB en más de 30 años en Gran Bretaña no ha hecho que la gente sea más feliz (Gasperg, 2004). La teoría del capital humano tampoco explica por qué la gente toma la decisión de invertir en educación, o en más educación, o bien de contradecir esta inversión. Tal y como explica Robeyns (2006), el capital humano no puede responder de los bienes no económicos de la educación, como que alguien quiera aprender poesía porque sí.

Además, ahora estamos en una época de crisis económica. Pero el hecho de centrarnos en los resultados del capital humano y en los impulsores de las políticas de mercado en educación superior a lo largo de varias décadas no nos ha equipado para evitar este resultado ni ha eliminado las desigualdades en el centro de la sociedad. Seguimos lejos de la solución de conflictos renacientes basados en identidades, culturas y religiones impugnadas.

\section{Pedagogía de reforma y equidad "débil”"}

No obstante, en esta era de planteamientos desenfrenados acerca del capital humano, encontramos pedagogías sustentadas por la preocupación de la equidad para el aprendizaje de los estudiantes y la aplicación del acceso y la participación de los estudiantes en el proceso de aprendizaje. Por ejemplo, la relación entre investigación y enseñanza y las culturas pedagógicas en cuestión se considera central en lo que debe ser una universidad. Aun así, el hecho de tomar este nexo de investigación y formación como ejemplar, sugiere que estas culturas pedagógicas están incorporadas en versiones débiles de equidad. Brew (2006), en su texto escrito recientemente, contribuye en cierta medida en el tratamiento de los propósitos más amplios de la educación superior en el sentido que considera la relación entre la investigación y la formación integral para el desarrollo de una "nueva" educación superior basada en un planteamiento pluralista e "inclusivo" para comprender la relación entre investigación y formación. Brew afirma que este tipo de educación superior estaría basado en investigaciones y sería esencial para futuros imprevisibles y para tratar algunos de los problemas más importantes del mundo (ibíd.: xiv), como la pobreza mundial. Plantea 
que para conseguirlo todo depende, en gran parte, de una educación basada en investigaciones, como si éstas generasen por sí solas estos compromisos e intereses. Con todo, el riesgo es que una filosofía de investigaciones críticas podría ser simplemente una que promoviera el individualismo y los valores de mercado, tal y como nos recuerdan los valores y las actitudes de muchos graduados. Para ser justos, Brew reconoce que la enseñanza incluye la inculcación de disposiciones de ánimo como "mostrar interés y respeto". Apoya las "comunidades inclusivas de creación de conocimientos académicos" de estudiantes y profesores asociados en el reto de llegar a entender el mundo a través de investigaciones sistemáticas y tomas de decisiones en colaboración a la vista de las pruebas (ibíd.: 3-4). Se trata de objetivos loables para la educación superior. No obstante, no nos harán avanzar suficientemente si no articulamos a la vez un amplio conjunto de objetivos normativos acerca de la utilidad de llegar a comprender el mundo, los intereses de quien debería servir esta comprensión y el significado que ello tiene frente a los impulsores neoliberales. Las capacidades de la investigación podrían llevar fácilmente a formas técnicas de educación superior y de cambio.

Este método de aprendizaje fundado en investigaciones se puede conceptualizar como una pedagogía de reforma, con un marco débil de igualdad y unos compromisos débiles con la agencia moral y el desarrollo social y humano. Estaría caracterizado por fuertes compromisos con el aprendizaje de los estudiantes, y por compromisos más débiles con el apoyo de valores u orientaciones a la justicia. Los estudiantes se reconocerían por el hecho de tener una agencia en el aprendizaje, pero aparte de la agencia para el capital humano, no hay ninguna directriz clara sobre cómo evaluarla o valorarla, o bien sobre si alcanzaría la agencia como ciudadanos democráticos. Está establecido cómodamente con una concepción de la igualdad que prepara a los estudiantes para llevar a término un rol económico (Unterhalter, 2008). El planteamiento pedagógico reformista de Brew tipifica la ausencia de un discurso transformista en gran parte del trabajo en pedagogía de la educación superior, algo que omite la pregunta profundamente importante de Luke (2006: 5): “¿Qué son las pedagogías para el ser humano?", ni tampoco trata la llamada educativa de Barnett (2007: 153) para que un estudiante "sea diferente en el mundo". 


\section{Pedagogía de reforma y equidad "fuerte"}

¿Sería útil tener una mayor claridad de la forma de entender la justicia? Rawls (2001) sostiene que como seres humanos tenemos una capacidad para conocer la justicia y también para la concepción de lo que es bueno, y para revisarla y modificarla si contamos con una buena razón para hacerlo. La justicia nos proporciona una base de principios para decidir qué distribuimos, a quién y por qué motivo tan solo para disposiciones sociales y para el diseño de instituciones. La justicia también describe las obligaciones que tenemos los unos frente a los otros (Brighouse, 2004).

Al conceptualizar las características de una pedagogía de praxis, se me presenta la idea de que la justicia se debería entender como la primera virtud de una sociedad, de modo que los problemas sociales y educativos surgen del vacío entre la realidad educativa y las exigencia de la justicia (Strike, 1989). Para resolver contenciosos o decidir en acciones morales nos dirigimos a los conceptos de justicia (y no de investigación), de modo que "las apelaciones a la justicia realmente marcan una diferencia en el modo en el que se resuelven los contenciosos o en el tipo de políticas que se adoptan" (ibíd.: 18). En este caso, nuestros conceptos de justicia en relación con la pedagogía tienen cierta importancia: ¿cuáles son las características de los estudiantes que esperamos formar? ¿Cuál es el papel de la educación superior en relación con la sociedad? ¿Cuál es el papel de la educación superior en la distribución de los bienes y los servicios que producen la educación y la sociedad? (ibíd.).

Esto nos sigue remarcando la importancia del trabajo de fomento del bienestar para que el trabajo aumente las oportunidades de seguridad ontológica de las personas en una economía de alto riesgo que cambia rápidamente. De aquí la importancia de las libertades reales para hacer realidad los proyectos para conseguir una vida y un trabajo decentes en la sociedad, que requiere un equilibrio delicado entre los objetivos económicos y los sociales y el progreso social. Tal y como escribe Saláis (2004: 283): "El trabajo cambia, exige flexibilidad y autonomía; su práctica plantea la cuestión de las libertades efectivas y contradice la lógica de la subordinación". Por lo tanto, educar para el trabajo no conlleva una problemática intrínseca; lo que es problemático es la forma limitada de entenderlo y la forma en la que estas conceptualizaciones limitadas se 
traducen en versiones de equidad empobrecidas o "débiles" de las pedagogías que se basan en las habilidades y las de formación.

Strike (1989) sugiere que la diferencia está entre una concepción de equidad que pasa por formarse para un trabajo y otra que prepara a los estudiantes para entender el mundo donde viven, a la vez que se forman para un oficio. Este último apoyo de equidad requiere a los estudiantes desarrollar sus capacidades para comunicarse racionalmente y tomar decisiones de forma autónoma, sus capacidades de ciudadanía y justicia, y las de realizar actividades significativas junto con otros. También puede solicitar una atención particular a la estructura de las disciplinas (como historia, política y biología), porque proporcionan espacios únicos para el desarrollo de un discurso racional basado en las formas estándar y arguméntales de estas tradiciones disciplinarias y adjudicado para ellas (Strike, 1989). Esto también nos llevaría más allá de las preocupaciones más complacientes respecto a la relación de las disciplinas con el nexo de investigación y formación. Un planteamiento alternativo exigiría un concepto en el que la educación no sirva únicamente para el crecimiento económico, sino también para la creación de una comunidad democrática y para contribuir con más justicia a la sociedad y al mundo.

\section{Dos características centrales de una pedagogía de praxis}

Todavía más interesante resulta el razonamiento de Barnett (2005b: 94), que afirma que la investigación no se ve solamente retada por un mundo incierto y "supercomplejo", sino que además también se debe orientar la formación a la producción de capacidades humanas: cualidades y disposiciones". La enseñanza debe realizar un "giro ontológico" del saber al ser, en el que los profesores tengan en cuenta a los alumnos "como seres humanos y no como seres con conocimientos", de modo que los estudiantes tengan una posibilidad abierta de "introducirse en una nueva forma de ser" (Barnett, 2007: 1). Sugiere que la ontología triunfa pero no sustituye la epistemología. En la enseñanza universitaria se debería tener en cuenta tanto el saber como el ser. Barnett sostiene que un mundo de incertidumbre y cambios no sólo plantea retos de currículo relacionados con el saber y con las acciones correctas a emprender, sino que también nos reta como seres humanos en este mundo. ¿Cómo me comprendo a 
mi mismo? ¿Cómo puedo orientarme? ¿Qué posición tengo en el mundo? ¿En qué me convertiré como ser humano como consecuencia de lo que experimento como alumno? Si el nexo de investigación y formación no consigue tratar este tipo de cuestiones, deberíamos preguntarnos qué sentido tiene este debate.

En respuesta, esbozo dos características centrales de un planteamiento pedagógico de praxis para comprender el valor de un nexo de investigación y formación.

\section{Aptitudes como base para la justicia social}

Una pedagogía de praxis no estaría sólo basada en oportunidades económicas, sino también en los conceptos más amplios del desarrollo humano, el bienestar y la dignidad para insertar un idioma de "aptitudes humanas" (Sen 1992, 1999, 2003; Nussbaum 1997, 2000) en el espacio de la investigación y la formación. Esto sería fundamental, no se podría negociar y representaría la base de la equidad y la justicia. Todos los estudiantes deberían disponer de la oportunidad de formar las aptitudes que se harían posibles mediante una educación superior igualitaria. El punto central de la noción de aptitud es el hecho de concebir que una persona pueda llegar a desarrollar, a través de su educación, una comprensión razonada de los hechos valorados de ser y actuar. Se trata de un argumento potente para formas educativas a través de las cuales un individuo puede explorar su propio concepto sobre qué tiene razón de valorar. Si la expansión de aptitudes es un objetivo normativo importante, la educación superior supone una parte de la expansión de la capacidad de ser educado y también de la capacidad de realizar elecciones valoradas en otras esferas de la vida (Unterhalter et al., 2007). Por lo tanto, Sen sostiene que: "La capacidad de ejercer la libertad puede depender directamente, hasta cierto punto, de la educación que hemos recibido y, por lo tanto, el desarrollo del sector educativo puede tener una conexión de base con el planteamiento centrado en las aptitudes" (Sen, 2003: 12).

Sen elabora dimensiones intrínsecas e instrumentales para la educación. Tener educación ya es un éxito valioso en sí mismo; pero la educación también ayuda a una persona a hacer muchas otras cosas valiosas como conseguir un trabajo; además, mejora la libertad para conseguir toda una serie de hechos 
valorados procedentes del hecho de ganar un sueldo. Aunque en el planteamiento de las aptitudes se considera útil contar con una base de capital humano para la educación, también es limitado. Sen (2003) no rehúsa rotundamente el capital humano; de hecho, ve sinergias en la medida que el capital humano y el planteamiento de las aptitudes están conectados con el papel, la agencia y las capacidades de los seres humanos. Pero Sen argumenta que centrarnos en el crecimiento económico no cuenta qué importancia tiene este crecimiento o para qué sirve la riqueza. Por lo tanto, la educación no se debería centrar sólo en el capital humano y en la "utilidad" de los seres humanes excluyendo las finalidades no económicas también valiosas y las interpretaciones más expansivas de lo que es valioso en las vidas humanas. Así, para Sen, la dirección de la política actual de educación superior en el Reino Unido sería problemática, igual que lo sería un nexo de investigación y formación en el que no se hablase del valor de las vidas.

Para Sen $(1992,1999)$, las aptitudes comprenden las oportunidades reales, es decir, las libertades sustantivas que tienen las personas para hacer y ser lo que valoran ser y hacer. Pero se debe entender que las aptitudes no son lo mismo que las habilidades o las aptitudes internas (Burchardt y Vizard, 2006). Eso haría que el punto central fuera el éxito o el fracaso individual, mientras que el planteamiento de las aptitudes nos dirige a los acuerdos sociales (por ejemplo, las condiciones pedagógicas o los propósitos normativos de las universidades) que permiten o disminuyen la formación de aptitudes.

El desarrollo humano consiste en ampliar el conjunto de aptitudes a partir del cual todos los alumnos toman decisiones sobre su vida y su carrera, a través de la "supresión de distintos tipos de falta de libertades que dejan a las personas pocas elecciones y pocas oportunidades de ejercer su agencia razonada" (Sen 1999: xii). Los seres y las actividades de valor, o los funcionamientos, son constitutivos del ser humano; una aptitud es un funcionamiento potencial. Los funcionamientos pueden incluir entablarse en discusiones con coetáneos, pensar de forma crítica sobre la sociedad, estar informado, tener una posición ética, tener buenas amistades, ser capaz de comprender una pluralidad de perspectivas en un tema, etcétera. El desarrollo educativo en estos términos implica la ampliación de las aptitudes humanas y las capacidades para escoger una buena vida de bienestar entre distintas alternativas. La capacidad de elección 
entre alternativas serias ya implica una "característica valiosa en la vida de una persona" (Sen 2008: 8). El centro de atención está situado en lo que importa a la gente, en las cosas importantes de la vida de las personas, en lo que realmente pueden ser y hacer, en la formulación, la elección y la búsqueda de sus objetivos.

\section{i) Aptitudes funcionales como evaluadores fuertes}

Pero aunque distintas aptitudes pueden constituir un conjunto de aptitudes de un individuo y se pueden formar mediante la pedagogía (por ejemplo, la aptitud de pensamiento crítico, o la de imaginación, o la de voz), el planteamiento de aptitudes de Sen tiene poco que decir sobre qué aptitudes se deberían promover a lo largo de la educación superior, o sobre el motivo por el cual los funcionamientos (consecuciones) son tan importantes en la educación como las aptitudes. Sen tampoco explora el contenido de una agencia, de modo que "el individuo que sabe escoger y razonar" es central, "pero con contenidos más específicos sobre el hecho de ser humano" (Des Gasper, 2002: 451). La formación de la persona solo se trata por encima (ibíd.: 451). En educación, esto no es suficiente. Nussbaum (2000) es más útil en este caso, puesto que proporciona una lista de 10 aptitudes humanas centrales, muchas de las cuales tienen que ver con la educación, y en mi trabajo he hecho y rehecho esbozos sobre qué pueden constituir las aptitudes más importantes de la educación superior (Walker, 2006).

No obstante, no pienso analizar ni su lista ni la mía. En lugar de ello, quiero resaltar que para desarrollar una imagen de un ser humano rico y complejo, debemos pensar en las aptitudes que la educación superior debería fomentar y también que sería razonable esperar que los estudiantes ejercieran (Wolff, 2007).

Por este motivo considero útil a Charles Taylor (1985). Introduce una dimensión ética explícita (que se podría decir que no existe en el planteamiento de las aptitudes) a la elección mediante la conceptualización de los seres humanos como "evaluadores fuertes", que se autointerpretan y que pueden evaluar que algunos valores éticos o ideales o bienes son más importantes que otros. Desarrollar la aptitud de los estudiantes como evaluadores fuertes significa desarrollarlos como sujetos capaces de reflexionar acerca de sus valiosas finali- 
dades y de volver a examinarlos cuando se les reta a que lo hagan. Reflexionan sobre lo que tiene un significado más o menos ético en la interpretación narrativa de sus vidas. Pienso en una versión robusta de la capacidad de reflexión que permita a los individuos cambiar mediante la reflexión acerca de lo que más les importa, incluso en condiciones de coacción (Archer, 2003). Esta capacidad de reflexión es operativa e implica "cuestionarnos a nosotros mismos, mediante la clarificación de nuestras creencias e inclinaciones, el diagnóstico de nuestras situaciones, la deliberación sobre preocupaciones y la definición de nuestros propios proyectos" (ibíd.: 103). Significa reflexionar acerca de nuestros propios funcionamientos y objetivos valorados, y tener la agencia para llevar a término estos objetivos, o incluso cambiarlos.

Al fin y al cabo, queremos animar nuestros alumnos a decidirse a actuar de forma valiosa y que valga la pena, y no de forma trivial o peor. Por lo tanto, ser un evaluador fuerte es un tema de conocimientos y capacidades, y también una "aptitud moral" (Van Staveren, 2001).

Esto declina más hacia un discurso ético y de praxis que hacia una cuestión más reformista acerca de cómo se impulsa una cultura de investigación o como se debe desarrollar un aprendizaje activo o de investigación y aprendizaje. La calidad en el aprendizaje de los estudiantes requeriría integrar el aprendizaje del tema y el desarrollo de un juicio reflexivo acerca de qué hace que la vida sea buena para una persona, es decir, su bienestar y la agencia. Podemos discutir que las pedagogías se deberían evaluar en términos de si las libertades sustantivas que tienen los estudiantes se han ampliado de forma que puedan hacerse y ser ontológicamente seguras, con las aptitudes para realizar elecciones bien razonadas, críticas y reflexivas en un mundo incierto sobre qué hace que la vida sea buena para ellos. Esta visión de la agencia moral también vería el hecho de pertenecer a la comunidad como central para el ser, y no instrumental como parece ser en el caso en Sen (Des Gasper, 2002). Al analizar el aprendizaje y la pedagogía, deberíamos buscar pruebas de ejes de uno mismo, los otros y la sociedad. Deberíamos subestimar la importancia del énfasis en el desarrollo de cada persona en el planteamiento de las aptitudes: esto es fundamental para la educación superior, pero también supera al individuo como participante en procesos políticos y no sólo como receptor pasivo. 
Para tomar un ejemplo de selección de aptitudes profesionales, en mi proyecto actual sobre educación profesional en Sudáfrica, desarrollamos de forma participativa un esbozo de aptitudes profesionales de desarrollo humano, y los funcionamientos que fluyen de ellas (véase Walker, et al., 2008). Cada uno tiene implicaciones para los planteamientos pedagógicos. En primer lugar, consulté la lista de Nussbaum, pero también hablé con profesores universitarios y estudiantes implicados en la educación profesional. Tenemos la idea de Taylor de los evaluadores fuertes y los hemos presentado así:

\section{Aptitudes profesionales de desarrollo humano Objetivos profesionales y calidades como funcionamientos}

Licenciados fuertes que son evaluadores

- Tener la aptitud del razonamiento práctico (llevar a término la acción correcta, en el momento adecuado y en las circunstancias acertadas).

- Ser capaz de evaluar algunos valores éticos o ideales o bienes para ser más importantes que los otros.

- Ser capaz de reflexionar y volver a examinar las finalidades valoradas, recorriendo a la teoría y los conocimientos académicos.

\section{Imagen 1}

Como la pedagogía y el desarrollo de los estudiantes están enmarcados ínstitucionalmente, y como hay dimensiones institucionales de desigualdad de aptitudes (que podemos denominar exclusión social), hemos empezado a desarrollar dimensiones institucionales e "indicadores" más específicos que señalen los acuerdos sociales y formen el desarrollo de aptitudes. El contexto institucional y los escenarios pedagógicos se deben mantener juntos. Uno de los ejemplos que hemos pensado desarrollar es este: 


\section{Dimensión}

Indicador

Conectividad

- La universidad está conectada con la sociedad y ésta, a su vez, está conectada a la universidad; los cambios en una influyen en la otra;

- Tanto los alumnos críticos como los intelectuales públicos;

- La investigación y la formación tratan de las "urgencias morales" de la ciudad, la región y el campo;

- El liderazgo "dice la verdad al poder".

\section{Imagen 2}

La primera función central de una pedagogía de praxis es, por lo tanto, que amplía las aptitudes de los estudiantes y sus logros de funcionamiento para ser y convertirse en evaluadores fuertes; las pedagogías de praxis también tienen implicaciones en los contextos y los acuerdos institucionales.

\section{ii) La aptitud funcional del razonamiento crítico}

Si seguimos reflexionando sobre qué aptitudes funcionales pueden ser especialmente significativas en educación superior, se debe tener en cuenta que esta educación superior normalmente supone un punto a favor del desarrollo de las capacidades de pensamiento crítico de los estudiantes. No obstante, en las condiciones actuales, a menudo parece que estas competencias deban mejorar la capacidad para encontrar trabajo en una economía del conocimiento, y no la capacidad de ser educado. Simons (2006) lo ejemplifica en su informe sobre el hecho de ser educado mediante la investigación. Tal y como explica Simons (2006), en el contexto europeo, el punto de partida para las políticas no es la antigua perspectiva de Humboldt sobre el potencial edificador de la investigación académica, sino las necesidades económicas de la sociedad. La investigación se reformula como otro "método" de enseñanza. Pero Simons argumenta que la reformulación de la educación a través de la investigación como un conjunto de competencias que se deben lograr disminuye la erudición y la búsqueda o el deber de la verdad. Tal y como pregunta Simons (2006: 43), ¿existe todavía un 
"deber académico o una orientación normativa en investigación que permita reflexionar acerca de la 'educación a través de la investigación’ y que sea diferente de la reflexión que crearon las necesidades de la sociedad del conocimiento y la operacionalización de la investigación como 'método de investigación'?".

Papestephanou y Angelí (2007) explican el problema. Señalan dos discursos diferentes que forman el pensamiento crítico, que tiene tendencia a aparecer de forma prominente en listas de competencias o habilidades de investigación deseables. Por un lado, tenemos el paradigma de las habilidades (que se pueden encontrar en discursos y prácticas de habilidades clave, habilidades genéricas, habilidades transferibles y atributos graduados) incorporadas a la agencia, el tecnicismo y la racionalidad intencionada de Habermas, que es "relevante para los papeles del cliente y el consumidor de servicios y bienes, y no para el participante activo en la posible transformación de la esfera pública" (ibíd.: 609). La idea es optimizar los resultados; pero en el caso de los resultados del capital humano de la educación superior del Reino Unido, estas finalidades no están abiertas a críticas. Lo que cuenta es la "efectividad, los resultados y el rendimiento" (ibíd.: 607). No se cuestionan las finalidades y el valor, ni tampoco se revisan los objetivos; de este modo, la educación superior, y su petición fundamental de fomentar el pensamiento crítico, se ve capturada por el proyecto neoliberal, a la vez que irónicamente sigue pareciendo servir sus propios valores y propósitos para desarrollar el "pensamiento de alto nivel".

Pero Papestephanou y Angelí también argumentan que "hay un excedente de pensamiento crítico que no se puede canalizar en el debate sobre las habilidades", algo que constituiría la educación a través de la investigación. Utilizan el concepto de Habermas de racionalidad comunicativa, que está orientado al potencial humano y a las acciones para la comprensión mutua, el diálogo formativo, el autoanálisis y la transformación de las finalidades para que "los objetivos no sean sólo para ser logrados o aproximados; lo más importante es que se verifiquen en introspección, y mejor si es en deliberación" (ibíd.: 609). De este modo, el pensamiento crítico es moralmente pertinente (ibíd.:609). Una visión del pensamiento crítico como una racionalidad comunicativa discutiría que "un pensador crítico no puede ser sólo el que lleva a término una acción correctamente, sino principalmente alguien que considere y que, cuando sea necesario, cuestione la adecuación o la relevancia moral de la acción” (Papestephanou y Angelí 2007: 608). 
Tal y como sostiene Strike (1989), aunque el pensamiento crítico es una virtud democrática, la justicia igualitaria exige más que la capacidad de razonar correctamente. También requiere la capacidad de la escucha dialógica, de la interpretación justa de las perspectiva de los otros, del desacuerdo respetuoso y de la participación correcta en las complejidades de la toma de decisiones democrática. La traducción de la educación mediante la investigación en competencias "tiene tendencia a olvidar que el potencial edificador de la investigación es algo que (todavía) no se puede dominar como un conjunto de competencias técnicas" (Simons, 2006, pág. 47). Aunque el hecho de que los empresarios quieran licenciados con las capacidades para llevar a término investigaciones no sea un problema intrínseco, debemos entenderlo de forma crítica y debemos comprender por qué esto se ha convertido en una preocupación política en el Reino Unido.

La segunda característica central de una pedagogía de la praxis no implicaría sólo las capacidades de pensamiento crítico, sino también la educación mediante la investigación para que los estudiantes desarrollen la aptitud de razonamiento comunicativo y de agencia moral en sus deliberaciones y mediante éstas.

\section{Impulso de los derechos pedagógicos}

En segundo lugar, una parte integral de una pedagogía de praxis sería que los estudiantes tuvieran "derechos pedagógicos" específicos (Bernstein, 2000) $\sin$ los cuales no desarrollarían las aptitudes funcionales para contar con una participación igual y efectiva en la sociedad para vivir bien en una democracia (Walker, 2006). Las aptitudes (mínimamente por ser evaluadores fuertes y razonadores críticos) asegurarían, implementarían y evaluarían estos derechos más allá de la política y la retórica (Nussbaum, 2000). Bernstein aboga por los tres derechos integrados que abarcan a uno mismo, los otros y la sociedad y que tienen el apoyo de asunciones de obligaciones éticas más allá del interés de uno mismo por defender o promover los derechos pedagógicos de cada persona (Bernstein, 2000; Sen, 2004). Los derechos en cuestión son: la "mejora", que implica una comprensión crítica y una visión de nuevas posibilidades y que es la clave de la formación de la confianza y la agencia. 
El segundo derecho es la "inclusión", el derecho a ser incluido socialmente, intelectualmente, culturalmente y personalmente, algo fundamental para la "communitas". La tercera es la "participación" en la formación y la transformación de los resultados políticos ("práctica cívica"). Estos derechos serían integrales a las relaciones de intersujetividad pedagógica caracterizada por la dignidad humana y la realización de todo el potencial de cada persona al adquirir conocimientos. Las aptitudes "identificadas y privilegiadas en la formulación de los derechos en cuestión" (Sen, 2004: 321) se deberían ampliar mediante las pedagogías de investigación y formación para asegurar estos derechos.

El hecho de centrarnos en los derechos también nos dirige a los aspectos de libertad del proceso, incluidos los procesos pedagógicos. Sen tiene claro que, aunque la aptitud es importante para la evaluación del aspecto de oportunidad de la libertad, "no puede tratar de forma adecuada con el aspecto de proceso de la libertad, porque las aptitudes son características de ventajas individuales, y se quedan cortas para darnos suficiente información acerca de la imparcialidad de la equidad en los procesos implicados, o acerca de la libertad de los ciudadanos para invocar y utilizar procesos equitativos" (ibíd.: 336), por lo tanto "el proceso a través del cual suceden las cosas también puede ser importante para evaluar la libertad" (ibíd.: 585).

Para quienes sostienen que las nociones de derechos humanos pedagógicos en educación y que las obligaciones éticas que nos deberíamos enseñar los unos a los otros no son posibles en las condiciones neoliberales contemporáneas, les responderíamos, siguiendo Sen, que el hecho de que estos derechos no sean posibles o no lo sean completamente en las condiciones actuales, "no implica nada que se aproxime a la conclusión que no son derechos" (2004, pág. 348). Nos recuerda que la ausencia de estos derechos nos indica el trabajo necesario para cambiar las circunstancias políticas e institucionales generales. Además, afirmar que los derechos pedagógicos también son derechos humanos es afirmar que las instituciones educativas que no los promuevan o protejan de forma práctica son defectuosas.

Así, la segunda característica central de la pedagogía de la praxis serían los derechos pedagógicos específicos. 


\section{Pedagogía de praxis}

Si unimos estas dos características centrales de aptitudes funcionales como evaluadores fuertes y razonadores críticos y de derechos pedagógicos, nuestras evaluaciones (y nuestro desarrollo) de pedagogías y calidad e igualdad nos dirigirá al aprendizaje de los estudiantes y los logros de este aprendizaje. Los derechos y la especificación de aptitudes valiosas actuarían junto con el planteamiento de las aptitudes para generar personalidades ricas en lugar de la versión más ligera del trabajo de Sen (Des Gasper, 2002). Presentan un esbozo que va más allá de la simple elección (porque podemos realizar elecciones equivocadas y porque lo que escogemos es importante) para indicar los parámetros fuertes pero imprecisos (Nussbaum, 2000) de una buena vida para uno y para los otros (Des Gasper, 2002).

Paula, una estudiante de último año de historia en una universidad inglesa donde se trabaja con el planteamiento de investigación, comenta los éxitos que impulsa esta pedagogía de praxis describiendo algunos de sus funcionamientos valorados:

Me hizo ser más consciente de mi punto de vista y del modo de ver las novedades y cosas como las asunciones que hago; vengo de un entorno de gente blanca de clase media, de una ciudad donde no hay tensión racial porque sólo hay gente blanca y de tal modo, que es muy fácil hacer asunciones o tener opiniones que nunca tienes que poner a prueba porque estás rodeado del mismo tipo de gente que tú; creo que esto también forma parte del hecho de venir a la universidad; este curso me ha hecho reconsiderar muchas cosas y pensar en mis prejuicios y mis estereotipos y todo eso. Creo que esto me ha dado más confianza en un tipo de debates. Los debates solían ser bastante intensos en mi escuela, pero todo el mundo tenía la misma opinión... Dicho esto, no tengo ni idea de qué es lo que quiero hacer... así que podría resultar difícil unir mis ideales con la realidad del mundo, no estoy segura... Pienso en cómo me ha hecho repensar mis prejuicios porque creo que significa mucho, o sea que los juicios y los prejuicios te forman, caracterizan en buena parte la forma de tratar con el mundo y la gente, la forma de leer, de interpretar y todo este tipo de cosas, el hecho de tener que mirarlo y retar a quienes se está retando (Walker, 2008: 36). 
En la clase de historia donde asistía Paula, la pedagogía del profesor estaba formada por los derechos pedagógicos: los estudiantes desarrollaban su confianza individual mediante una buena formación; participaban con los otros en la crítica y la formación del conocimiento, y desarrollaban como mínimo un sentido modesto de la agencia cívica. Peter Otto, su profesor, comenta la importancia de esta "práctica cívica" adecuada, y explica su propia práctica pedagógica:

No les enseño qué deben ser, de izquierdas o de derechas; está claro que tengo mis preferencias, pero no es mi tarea ni mi trabajo, de modo que no debería decir: "Esta es la perspectiva correcta del mundo". En lugar de ello, lo que intento enseñarles es: "Mirad, es difícil y a veces hay contradicciones que no se pueden solucionar fácilmente y hay que tomar una decisión. Esta decisión debe estar basada en diferentes opciones y cada decisión tiene implicaciones morales y debéis saber que no podéis decir simplemente: 'mi forma de vivir la vida es la mejor y la única, de modo que es fantástico vivir así y no soy responsable de las consecuencias"” (ibíd.: 32).

Esta estudiante captura la formación de la agencia cívica, aunque sea modesta, cuando dice: "No creo que haya cambiado en un sentido dramático, pero me ha hecho ser más consciente de la forma de ver el mundo... Creo que, si eliges la opción correcta, es algo que podría marcar una diferencia, no sé".

\section{Conclusión}

Una pedagogía de praxis probablemente debería reconocer que en los tiempos actuales, y de forma estratégica, las universidades deben responder a las oportunidades del mercado. Pero también están comprometidos con el desarrollo de los estudiantes y con la sociedad, basándose en la formación responsable y la investigación verdadera. Los licenciados a quienes educan ganan individualmente, pero también son portadores del bien público mediante los conocimientos que han adquirido. Esto debe estar unido a un espíritu crítico y a unos valores democráticos que la pedagogía de praxis quiere cultivar como integrales para obtener conocimientos. De este modo, no sólo se adquirirían los conocimientos para llegar a ser científico o trabajador social, ingeniero o histo- 
riador, sino que también se formaría una perspectiva moral sobre cómo ejercer estos conocimientos para mejorar las vidas en las sociedad.

Nussbaum (2006) sostiene que la educación universitaria no se debería interpretar sólo como una simple producción de habilidades técnicas, sino también, y lo que es más importante, "como una atribución de poderes general de la persona mediante la información, el pensamiento crítico y la imaginación". Une implícitamente la democracia y la educación porque: "Los ciudadanos democráticos también pueden no pensar críticamente acerca de lo que sienten, pasando la rabia, el miedo y el poder por encima de la razón" (pág. 3). La mente democrática es una mente humana (y como seres humanos, somos imperfectos; cada uno de nosotros personifica la oscuridad y la luz), puede ser "negligente, propensa al pensamiento precipitado e irracional" (pág. 3). Necesitamos una educación que "cultive los seres humanos y su humanidad, en vez de producir generaciones de máquinas útiles" (pág. 15). Como mínimo, todavía se debe mantener un debate, incluso contra la ideología del mercado, acerca de los propósitos normativos y los resultados de las universidades. También podemos seguir produciendo licenciados en educación superior con habilidades tecnológicas, científicas, vocacionales y de investigación, a quienes a la vez les falten facultades críticas, imaginación y estar abiertos a la participación en la vida pública democrática. La pedagogía de praxis esbozada en este capítulo reta esta visión y ofrece un marco de trabajo para imaginar una alternativa en relación con las funciones principales de las universidades de investigación y formación y a sus interconexiones pedagógicas transformadoras.

\section{Nota}

1. Las entrevistas formaron parte del proyecto "Ontology, identity formation and lifelong learning outcomes: theorising the relationship between discipline-based research and teaching", financiado el 2006-2007 con una beca de investigación de la Academia de Educación Superior (HEA). Agradecemos gratamente el apoyo financiero de la HEA. 


\section{Referencias bibliográficas}

\section{Textos}

Barnett, R. Realizing the University in an Age of Supercomplexity. 2000.

(Buckingham, SRHE/Open University Press) Barnett, R.(Ed)(2005a) Reshaping the University. New Relationships between. Research, Scholarship and Teaching. (Maidenhead, SRHE/Open University Press).

Barnett, R. Recapturing the Universal in the University, EducationalPhilosophy and Theory, 37 (6) p. 785-797. 2005b.

Barnett, R. A. Wil! to Learn. Being a Student in an Age of Uncertainty (Maidenhead, SRHE/Open University Press). 2007.

Bernstein, B. Pedagogy, Symbolic Control and Identity. Londres: Routledge. 2000.

Brew, A. Research and Teaching: Beyond the Divide. Nueva York: Palgrave. 2006.

Brighouse, H. Justice. Cambridge: Polity Press. 2004.

Brighouse, H. The globalization of higher education and a professional ethics for academics. Conferencia plenaria presentada en el congreso "Learning Together- Reshaping higher education in a global age". Londres, 22-24 de julio de 2007.

Gasper, D. Subjective and objective well being in relation to economic inputs: puzzles and responses. Informe presentado en el taller sobre Aptitudes y felicidad, St. Edmunds College, Cambridge. Marzo de 2004 GU1MI. 2008. Higher Education in the World. Nueva York: Palgrave MacMillan. 2004.

Harvey, D. A. Brief History of NeoÜberalism. Oxford: Oxford University Press. 2005.

Jenkins, A.; Breen, R.; Lindsay, R. y A. Brew. Re-shaping Teaching in Higher

Education: Linking Teaching and Research. Londres: RoutledgeFalmer / SEDA). 2003.

Jenkins, A.; Healey, M. y R. Zetter. Linking teaching and research in the disciplines. York: Academia de Educación Superior. 2007. 
Luke, A. Introducción de la editorial: Why Pedagogies?, Pedagogies, en International Journal, 1 (1) p. 1-6. 2006.

Mclean, M. Pedagogy and the University. Londres: Continuum. 2006.

McLean, M. y H. Barker. Students making progress and the research-teaching nexus debate, Teaching in Higher Education, 9 (4) p. 407-419. 2004.

Nussbaum, M. Cultivating Humanity. A Classical Defence of Reform in Liberal Education. Cambridge: MA, Harvard University Press. 1997.

Nussbaum, M. Women and Human Development. Cambridge: Cambridge University Press. 2000.

Nussbaum, M. Education and Democratic Citizenship: Capabilities and Quality Education. Journal Óf Human Development, 7 (3) p. 385-396. 2006.

Papestephenou, M. y C. Angelí. Critical Thinking Beyond Skill, Educational Philosophy and Theory. 39 (6). p. 604-621. 2007.

Rawls, J. Justice as Fairness. A Restatement. Cambridge: MA: The Belknap Press. 2001.

Robeyns, Ingrid. "Three models of education: rights, capabilities and human Capital", Theory and Research in Education 4 (1) p. 69-84. 2006.

Saláis, R. Incorporating the capability approach into social and employment Policies, en R. Saláis y R. Villeneuve (eds.) Europe and the politics of capabilities Cambridge, Cambridge University Press. 2004.

Sen, A. Inequality Re-examined. Oxford: Oxford University Press. 1992.

Sen, A. Development as Freedom. NuevaYork: A. Knopf. 1999.

Sen, A. Human capital and human capability, en Fukudo-Parr, S. y A. K. Kumar (eds.) Readings in Human Development. Nueva Deli: Oxford y NuevaYork: Oxford University Press. 2003.

Sen, A. "Elements of a Theory of | Human Rights", en Philosophy and Pubiic Affairs, 32 (4). p. 315-356. 2004.

Simons, M. "Education through Research" at European Universities: Notes on the Orientation of Academic Research, Journal of Philosophy of Education, 40. p. 31-50. 2006. 
Strike, K. Liberal Justice and the Marxist Critique of Education. Nueva York y Londres: Routledge. 1989.

Taylor, C. Human Agency and Language. Philosophical Papers 1. Cambridge: Cambridge University Press. 1985.

Taylor, P. "Introduction", en GUNI Higher Education in the World. Nueva York: Palgrave MacMillan. 2008.

Walker, M. Higher Education Pedagogies. Maidenhead: SRHE/Open University Press. 2006.

Walker, M. Ontology, identity formation and lifelong learning outcomes: theorizing the relationship between discipline-based research and teaching. York: Academia de Educación Superior. 2008.

Zamorksi, B. Research-Led Teaching and Learning in Higher Education Centro de investigación aplicada sobre educación superior, Universidad de Anglia del Este. 2000.

\section{Sitios web}

Kwiek, M. The social functions of the university in the context of the changing State/Market relations. The global, European Union and accession countries' perspectives. Disponible en línea, en $<\mathrm{http}$ ://www.cpp.amu.edu.pl/kwiek/> publications.html. Consultado el 30 de noviembre de 2006. 2002.

Envío 17 de junio/2010 - Aceptación 17 de noviembre/2011 